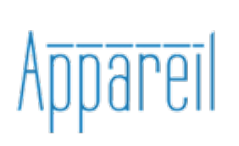

Appareil

$7 \mid 2011$

La transparence

\title{
De la transparence à la « disparence »: le paradigme photographique contemporain
}

\section{Muriel Berthou Crestey}

\section{OpenEdition}

1 Journals

Édition électronique

URL : http://journals.openedition.org/appareil/1212

DOI : 10.4000/appareil. 1212

ISSN : 2101-0714

Éditeur

MSH Paris Nord

Référence électronique

Muriel Berthou Crestey, « De la transparence à la « disparence » : le paradigme photographique contemporain », Appareil [En ligne], 7 | 2011, mis en ligne le 11 avril 2011, consulté le 30 juillet 2020. URL : http://journals.openedition.org/appareil/1212; DOI : https://doi.org/10.4000/appareil.1212

Ce document a été généré automatiquement le 30 juillet 2020.

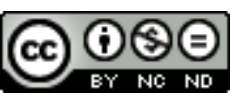

Appareil est mis à disposition selon les termes de la Licence Creative Commons Attribution - Pas d'Utilisation Commerciale - Pas de Modification 4.0 International. 


\title{
De la transparence à la « disparence » : le paradigme photographique contemporain
}

\author{
Muriel Berthou Crestey
}

1 Aujourd'hui, les dispositifs technologiques s'apparentent à des interfaces avec le monde permettant une exacerbation du visible, comme un écho au panoptique de Bentham. Il faut tout voir et tout montrer, au risque de créer des «habitus perceptifs » qui ne permettent plus de distinguer l'image saillante du réel. Là où la camera obscura dissimulait le processus de révélation de l'image, l'écran numérique permet au contraire la prévisualisation de l'image latente. Le système de la «boite noire » littérale est devenu le médium de la surinformation visuelle. Notion labile, la transparence s'apparente à une "figure [imposée] des temps post-modernes ${ }^{1}$ », permettant l'avènement d'un nouveau régime de visibilité photographique. L'exposition Lumière, transparence, opacité (Nouveau Musée national de Monaco, 2006) a permis de révéler les liens ontologiques qu'entretiennent ces notions avec le dispositif photographique, proposant une mise en abyme de cette "écriture avec la lumière ». Sur le plan sociologique, le lancement en 2001 de L'International Campaign for Media Transparency sous l'égide de l'IPRA est symptomatique de ce phénomène. Car en tout état de cause, le fantasme de l'absolue transparence est prégnant dans notre civilisation de l'image où règnent les "régimes esthétiques " axés selon Jacques Rancière sur une visibilité exacerbée et autres « régimes de lumière » deleuziens. Le monde nous est donné à voir " photographiquement ». Sous la surface apparente, il nous faut chercher à distinguer, comme dans les images d'Epinal, ce qui s'y dissimule. Or, le dispositif photographique permet de les révéler, à la lumière de l'énigme de la nouvelle d'Edgar Poe, La Lettre volée. Pour pénétrer les mystères de cette "doublure d'invisible » dont parle MerleauPonty, il faut alors s'interroger sur ce dispositif singulier qui consiste à voir à travers un objectif transparent. Réfléchir sur la transparence photographique contemporaine nécessite de revenir sur l'opacification progressive de ses modes de réception. Comment s'effectue ce passage de la transparence à la disparence photographique? Pour répondre à cette question ontologique, il s'agit d'aborder dans un premier temps 
les débats cristallisés autour de la transparence de la photographie, amenant ensuite à considérer un renversement de structure, à savoir une prise en compte de l'existence des photographies de la transparence. Ainsi affirmerons-nous le point de vue d'une réflexivité de l'image photographique envisagée pour elle-même, permettant ainsi, un instant, d'arrêter le regard.

\section{La transparence de la photographie}

\section{1. En toute transparence}

2 "Quoi qu'elle donne à voir et quelle que soit sa manière, une photo est toujours invisible : ce n'est pas elle qu'on voit ${ }^{2}$ " écrit Roland Barthes dans La Chambre claire. Pour le sémiologue, le médium photographique est celui de la transparence absolue, capable de retransmettre une image dans la plus pure humilité, en s'effaçant complètement du registre de la perception. C'est un témoignage direct qui joue, selon lui, le rôle de simple transmetteur d'informations. Dans cette perspective, le médium photographique se laisse traverser par le regard. Il fait office de médiateur. Aussi n'estce pas un hasard si l'auteur de ce texte publie, seize ans plus tôt, sa Rhétorique de l'Image dans laquelle il détermine la grammatologie visuelle des images publicitaires pouvant être envisagées comme autant d'«images-vitrines», soient des clichés dont la matérialité échappe constamment au regard, uniquement concentré sur le message véhiculé. L'aspect hétéroclite de ces objets d'études témoigne en réalité d'un point de convergence autour de la notion d'image transparente comme paradigme. Transférant le vocable linguistique au registre iconographique, la "photographie transparente " pourrait être définie comme une représentation lisible au premier coup d'œil, avec la fulgurance de l'évidence ${ }^{3}$. Une image comme un mot que tout le monde comprend. Lorsque Récanati marque la dichotomie entre locution transparente et opaque, il insiste de la même façon sur la valeur sémantique du mot dit «transparent» et sa propension à effacer sa valeur de signe au profit de ce qu'il représente ${ }^{4}$. Raisonner en termes de transparence revient donc à penser en termes de contingence. En 1980, Roland Barthes considère alors que l'intérêt d'une image photographique réside dans le primat accordé au référent. L'approche barthésienne de la photographie associe la notion de transparence à une fonction de transmission. Ainsi rejoint-elle les débats qui lui sont contemporains portant sur la théorie photographique de l'Index.

3 Déployant une pensée "à partir de la photographie et non pas sur elle ${ }^{5}$ », Rosalind Krauss élabore sa théorie photographique dans le climat d'effervescence qui accompagne l'émergence de la revue October en 1976, considérée comme un véritable " coup d'état ${ }^{6}$ ». Le projet fondamentalement pluridisciplinaire de la revue contribue à définir la photographie vis-à-vis des spécificités du médium. Rosalind Krauss y voit alors l'incarnation évidente de la notion d'empreinte restituant avec fidélité les objets du réel qui s'y impriment. Envisagée comme trace émanant du référent qu'elle désigne, la photographie se laisse traverser de part en part en fonction du sens de l'objet qu'elle donne à voir. Rosalind Krauss en vient alors à proposer sa théorie de l'Index élaborée à partir de Charles Peirce :

À la différence des symboles, les index établissent leur sens sur l'axe d'une relation physique à leur référent. Ce sont les marques ou les traces d'une cause particulière et cette cause est la chose à laquelle ils réfèrent, l'objet qu'ils signifient ${ }^{7}$. 
Le contexte intellectuel dans lequel s'organise cette théorie d'une photographie comme image référentielle permet d'entrevoir son développement généralisé en France. Alors que Gérard Deledalle organise la reparution d'un choix de textes de Peirce intitulé Écrits sur le signe (1978), Patrick Modiano publie la même année son roman Rue des Boutiques obscures. Si la photographie s'affiche avec ostentation dans cette œuvre littéraire, c'est parce qu'elle se présente en tant qu'objet figurant l'identité d'une personne, sans qu'il soit question d'examiner sa propre dimension identitaire. Quant il s'agit de partager son regard sur des photographies jaunies par le temps, le protagoniste opère systématiquement le même geste : celui de retourner la photographie afin d'y lire le sujet de la représentation, de la personne, de la fête ou de l'expression de l'émigration qu'elle désigne. « Il me passait les photos de plus en plus vite et ne les regardait même plus $^{8}$ » explique le narrateur. La photographie est la trace évidente d'un moment couché sur le papier, au dos de l'épreuve. Sa faculté de transparence est telle qu'elle n'impose même plus de poser un instant les yeux sur elle. L'année 1978 est aussi marquée par la sortie du dossier spécial « Photographier » d'artpress, privilégiant alors la référence à Bourdieu ${ }^{9}$ (Un art moyen, 1965). Au-delà du jeu de mots, le titre porte la clarté de son message : la photographie procède d'un usage limpide. Appliqué à une réflexion sociologique, sa fonction relève une fois de plus d'un acte de restitution du visible. L'intérêt croissant porté à la photographie par le milieu institutionnel (ouverture du département " photo » du Centre Pompidou en 1981), en parallèle de son accroissement théorique, tend à en faire une « image-miroir » du monde à mesure que sa visibilité s'étend. Cette révolution épistémologique associe paradoxalement la transparence du médium à son excès de visibilité. C'est parce qu'on la voit trop qu'on ne la voit plus. Le survisible est générateur de l'invisible. Dès lors, son mode d'inscription sociétal fait l'objet d'un mouvement pendulaire, à cette période charnière : intervient d'abord un type d'invisibilité lié à l'essence du médium (traces), rejoignant celle associée à sa réception. Mais à mesure que l'une s'affirme, l'autre disparaît. Plus la photographie prétend être liée à un référent invisible, plus nous la voyons apparaître, partout, dans les institutions. Dès ce moment, un déclenchement se produit au sein du monde photographique en train de se constituer. Les vingt commandements de l'image transparente mis au point par Kendall Walton en 1984 font état de cette perception de la photographie en tant que nouvelle mimésis. Le jugement est alors sans appel: "Photographs are transparent: we see the world through them ${ }^{10}$ " écrit-il. Ainsi le philosophe propose une photographie de la pensée la plus communément admise dans la sphère théorique propre aux années 1980. Un déclic inédit venu d'outre-atlantique abreuve le continent de sa naissance.

5 Serait-ce la muse amusée ? Roland Barthes, cité par Rosalind Krauss comme une des références primordiales de Notes on the Index, devient lui-même le récepteur des théories dont il fut le géniteur. La parution de La Chambre claire constitue alors un acte de reprise quant à la théorie du référent. Faut-il voir une relation avec l'investissement massif dont elle fait l'objet de la part des musées dans les années qui lui succédèrent? On ne peut qu'observer ce qui a été. Il s'agit alors de faire retour sur le noème fondateur mis en place par Roland Barthes: le ça a été. Pourquoi parler de la photographie comme d'un ça, précisément? Une personnification, au contraire, paraîtrait possible. Une approche $\mathrm{du}$ « ça voir » de Barthes nécessite de décomposer les structures du noème. À quelle personne en parler? En choisissant l'indéfini, le 
théoricien semble contourner le problème de la transparence du médium comme s'il s'agissait d'un acquis. Et pourtant, il le pose en son cœur.

\section{2. La transparence opaque}

$1^{\text {re }}$ occurrence: «[La photographie] dit: ça, c'est ça, c'est tel! mais ne dit rien d'autre ${ }^{11}$ »

Dans cette proposition, la photographie est entièrement soumise à son rôle déictique, c'est-à-dire qu'elle se fond dans le référent qu'elle montre. Elle n'existe pas pour ellemême et fait figure de simple re-présentation. La troisième personne est niée. C'est une relation fermée qui est ainsi créée, c'est-à-dire un échange en cercle clôt, entre un locuteur (le référent) et un interlocuteur (le spectateur). Dans cette perspective, la photographie serait un lieu de passage au sein duquel elle n'aurait pas son mot à dire. Transmetteur, vecteur de l'intermédiaire, ce médium s'exprime donc à la forme impersonnelle, avec le pronom auquel la contraction confère une connotation familière : "ça». Cela consiste pour elle à montrer, mais non pas à se montrer elle : la photographie. Ainsi, Barthes l'envisage selon un principe tautologique : « une pipe est toujours une pipe, intraitablement ${ }^{12}$ " précise-t-il. Provoquant un passage entre deux pronoms démonstratifs, du «ça » au «ceci », alors pouvons-nous admettre que l'objet fidèlement ressemblant à un référent ne se confond pas avec lui. Ainsi, Magritte pourrait répondre à Barthes que : «Ceci n'est pas une pipe » quand bien même cela en aurait l'apparence, selon la formule du célèbre tableau surréaliste. Pourtant, ici, c'est le message véhiculé par la photographie qui est tautologique et non la photographie en elle-même. L'idée qu'elle puisse renvoyer à son propre dispositif n'effleure pas l'esprit de Barthes, à cet instant, puisqu'il ne lui octroie aucun rôle véritable hormis celui de refléter l'image de la réalité. Pensant davantage la photographie que le photographique, il parle de l'image à la voix passive, comme un ça a été «vu». Cela revient pour lui à distinguer trois régimes d'application photographique : «faire, subir, regarder ${ }^{13}$ ». Dans cette relation, l'Operator manipule; le Spectator fixe. Processus dynamique d'un côté, statique de l'autre. En regard d'un cliché, ce dernier peut avoir pourtant comme tentation de regarder ce qui est « $\operatorname{tu}^{14}$ », c'est-à-dire, ce qui n'est pas montré. Cependant, toute photographie est pour lui éteinte, sans réponse. Quelque chose a disparu. L'échange semble alors unilatéral, allant de celui qui regarde à ce qui est vu. Chez le sémiologue incrédule à la possibilité d'une "image vivante", cet acte porte en lui une "blessure ${ }^{15}$ ", là où nous percevons dans le même temps une réparation potentielle, favorisant une catharsis par le regard. Or, c'est la prise en compte du médium pour lui-même qui permet cette transformation de la vue. Pour être visible, la photographie doit être opaque. Alors qu'il perçoit l'image en terme de contingence, nous la pensons également en termes de réflexivité photographique, dépassant le champ de la photographie " pensive $^{16} »$ : l'image est à voir ; elle se voit, se regarde elle-même dans le reflet qu'elle renvoie. Son usage ne se limite pas à panser les plaies de la psyché du Spectator. Simplement, elle plaît. Et c'est cette perspective intellectuelle en miroir qui nous permet d'examiner d'autres portées envisageables pour le ça.

7 Certes, le postulat de départ admis par Roland Barthes relève d'une observation irréfutable : une photographie peut avoir pour rôle de montrer, de pointer le doigt vers une instance du visible (déjà aperçue ou non). Néanmoins, l'interprétation qui en émane se veut être l'objet d'une autre hypothèse de développement. Et si, au lieu de 
n'être qu'un passeur, la photographie était un jeu complexe de relations imbriquées comme un système de boîtes gigognes? Alors c'est à travers ses yeux que nous percevrions le visible et l'invisible, comme en camera subjective. Dans cette perspective, le photographique est capable de parler en son nom, en s'affirmant comme un «Je » à part entière. Il apparaît et s'exprime tour à tour au titre de son identité personnelle ou de sa faculté de médiation. Les codes propres à la réception s'opacifient à mesure que le dispositif se découvre. Le photographique intervient alors potentiellement comme une troisième personne dans le nouveau schéma de la perception où il s'agit de "refaire, activer, regarder » sans ordre prédéfini qui soit donné au photographe ou au spectateur. Tous deux occupent des positions intermédiaires entre les temps conjugués du regard.

$2^{\mathrm{e}}$ occurrence : «C'était comme si je cherchais la nature d'un verbe qui n'aurait pas

d'infinitif et qu'on ne rencontrerait que pourvu d'un temps et d'un mode ${ }^{17}$ "

8 Ce verbe qui n'en est pas un, Roland Barthes l'a cherché dans l'auxiliaire «être » non pour en faire l'étymologie (Heidegger) mais plutôt pour questionner la morphologie temporelle associée à toute photographie. Au sein du noème barthésien, le ça est un sujet. Il faut donc porter notre attention sur ce qui anime le terme principal de la phrase: le passé composé «a été». L'auteur développe cette formule selon laquelle «dans la Photographie, je ne puis jamais nier que la chose a été là ${ }^{18}$ ». Le noème est ainsi qualifié d'«Intraitable ». Pourtant, trente ans après cette affirmation, la plupart des œuvres s'accordent sur l'idée que la chose photographiée n'a pas été. Certains photographes en ont fait le sujet de leur recherche. Ainsi, Joan Fontcuberta tient lieu de spécialiste quand à ce démantèlement épistémologique, où la photographie devient l'objet de la reconstitution de Miracles... Dans cette quête d'une origine ontologique portant sur le médium photographique, Roland Barthes réfléchit sur son propre Ça, soit l'image invisible cachée dans l'ouvrage (le portrait de sa mère enfant). Si elle n'apparaît pas, c'est qu' « elle n'existe que pour [lui] ${ }^{19} "$, dans un espace inaccessible au lecteur, à savoir le ça envisagé au sens freudien, cet espace à la source de toutes nos représentations, installé depuis notre naissance comme une boîte noire de résonances avec le visible. Il se manifeste uniquement sous forme d'images.

Un individu, précise Freud, se compose ainsi pour nous d'un Ça psychique, inconnu et inconscient, auquel se superpose le Moi superficiel ${ }^{20}$.

Dépourvu ici de sa majuscule, le ça de Barthes n'a pourtant rien perdu de son sens, constituant une véritable réaction somatique de l'invisible, à l'instar d'une photographie activée chimiquement par le principe de bain révélateur. Néanmoins, il n'a guère franchi le pas. Le cliché est devenu domaine réservé. Par ce geste, il conforte cette affirmation de l'image transparente, susceptible d'en dire trop. L'inconscient est informulé. Il n'y a plus, selon lui, d'infinitif possible car la finitude a mis à mal cette opportunité de faire accéder le visible à l'invisible, la transparence à son opacité. Or, le plaisir des yeux procuré par la contemplation d'une photo pourrait représenter une façon de parer à une réalité perdue. Son image inversée (le passage de l'opacité de l'image à la transparence du mot) apparait alors comme un ultime recours pour envisager sa proposition. Car le photographique implique une prise de conscience de notre propre regard. En donnant à voir les surfaces invisibles de la réalité et les conditions de sa perception, il nous porte à regarder le monde tel qu'il se présente à nous, dans son immanence. Offrant à contempler un univers opaque, il influe sur nos représentations du réel et incite à les repenser à la lumière d'un autre langage. 
10 Si l'ouvrage de Roland Barthes participe d'un engouement pour l'image référentielle qui marque les années 1980, il donne également naissance à une théorie inversée (telle l'image photographique) : celle d'une photographie opaque. S'en suit effectivement un déferlement d'articles qui marqueront à plusieurs titres ce cheminement de l'index photographique à la photographie de l'index. Combien de fois a-t-on assimilé les images photographiques à ce qu'elles représentent? Serait-ce un phénomène de « ressemblance par contact ${ }^{21}$ »? Analysant «L'œuvre de Duchamp sous le paradigme photographique ${ }^{22}$ ", Georges Didi-Huberman revient sur le territoire de Rosalind Krauss et insiste sur l'ingénieuse fusion opérée par la théoricienne américaine entre sémiologie et phénoménologie, mettant ainsi en œuvre un principe de réflexivité :

Tressant avec promptitude la notion linguistique d'embrayeur et la catégorie peircienne de l'indice, Rosalind Krauss ouvrait là une perspective remarquable, presque une façon de voir ${ }^{23}$.

11 Une façon de voir, certes, mais qui repose avant tout sur ce que l'on ne voit pas : le référent, absent, voire la plaie. Retour sur les traces de l'ouvrage déjà convoqué, compilant la première décennie d'October, celui-là même où figure L'indice de la plaie absente ${ }^{24}$. Un objet est à décrire. Qu'y percevons-nous ? Rien, « On n'y voit rien », déjà 25 , en 1983. Ou plutôt: "Presque rien", comme le précise le premier intertitre d'ouverture ; "c'est-à-dire : on voyait déjà autre chose qu'un rien dans ce presque. On voyait donc bien quelque chose, à simplement le prévoir, ou le désirer ${ }^{26} »$. S'il n'y a rien à distinguer dans la photo, alors l'image reste le seul élément perceptible. Il fallut qu'un seul jour le médium photographique ait décrété qu'il fallut voir ici un visage, là, dans ces taches, pour que le suaire fût, à jamais, auréolé d'une nouvelle aura. Le contact de la trace (la photographie) a pris le pas sur la trace du contact (le corps du Christ). L'image, même, se voudrait contingente; mais le discours à son encontre est alors nécessaire pour la voir. Elle ne représente " presque rien » mais est un support pour asseoir la véracité de ce qui ne se voit pas. Dès lors, la transparence photographique s'opacifie au contact de ce bouleversement ontologique. Susceptible de montrer autre chose que le référent originel, elle acquiert une nouvelle visibilité. Dans son court texte sur «L'Énonciation photographique ", André Rouillé propose une étude des codes photographiques liés au style, en s'appuyant sur l'exemple de la photographie publicitaire. Fondant son approche sur la relation réciproque unissant le concepteur et le récepteur, il en vient à associer ce médium à un principe de " communication sociale » quant bien même on lui aurait reproché, bien souvent, son mutisme. C'est sur ces fondements qu'il aborde la notion d'image/signe à la fois

en tant que «chose représentée » supposée quasi-invisible et diaphane devant la chose signifiée pour être alors hypostasiée dans une fonction de document neutre et objectif [mais aussi], dans le second cas, [l'image photographique est pensée comme étant] opacifiée, elle devient l'objet propre de la considération et cesse cette fois quasiment de représenter pour se présenter elle-même $\left[. . .{ }^{27}\right.$.

Invisible, oui. Mais pour qui ? Avec cette question, Jean-Marie Schaeffer porte la pierre de touche à l'édifice de Krauss, entérinant ce passage de la transparence à l'opacité. Pour qu'une théorie de l'indice puisse avoir une efficacité, encore faut-il savoir de quel côté nous nous positionnons. Plus que de s'interroger sur la question locative - «Où regardons-nous?" - il s'agit de se demander «qui regarde » et "d'où regardonsnous?». La question de l'ontologie du médium est ainsi posée en préambule de l'ouvrage de Jean-Marie Schaeffer, l'Image précaire, Du dispositif photographique paru dès 1987 dans lequel il s'agit de remettre à plat cette hypothèse américaine à partir d'un 
postulat engagé dans l'action. L'approche n'est plus à la voie passive. Elle s'organise autour d'un préalable fondateur: toute image a d'abord un point de destination. Qui voit? Qui écrit ? La photographie ? Le narrateur est-il apparent dans cette approche en aval de l'image?

Je pars de l'idée que l'image photographique est essentiellement (mais non exclusivement) un signe de réception. Je soutiens donc qu'il est impossible de la comprendre pleinement dans le cadre d'une sémiologie qui définit le signe au niveau de l'émission ${ }^{28}$.

Ce basculement prônant une approche de l'image visible est corroboré par un autre positionnement décisif: il s'agit cette fois de réfléchir en termes de dispositif photographique. Désormais, l'image est dégagée de toute contingence. Elle est pensée pour elle-même. Certes, en 1987, il est encore question d'une "spécificité physicochimique de la production de l'image, donc de son statut d'empreinte » à définir, dans la lignée d'une sémiologie appliquée à la photographie. Pourtant, l'arché de la photographie et son arc de diffusion se confondent alors au sein du concept de projection, qui intègre le champ lexical de la photographie, alors même que nous parlions plus volontiers d'empreinte pour exprimer son action matérielle. Cette conception ouvre la voie à l'Acte photographique tel qu'il parait sous la plume de Philippe Dubois: entérinant une position de balancier entre l'index et la valeur de transformation photographique, l'auteur va à l'encontre $d u$ «poids du réel» traditionnellement attaché à la photographie pour lui associer au contraire l'agent d'une métamorphose. Pointant l'argument structuraliste pour dénoncer «l'effet de réel ", il revient sur l'idée d'une photographie codée. C'est dans cette perspective qu'il s'agit de se positionner, à la lumière des pratiques hétérogènes mises en place avec l'ère numérique, dans une approche réflexive de la photographie. Cela met en jeu un paradoxe : activant un degré supplémentaire de transparence (appliquée à la techné), le photographique opacifie son message. La transparence opaque ainsi générée ne perçoit plus le référent, en partie effacé. Il s'agit moins de voir ce qui est représenté que de s'interroger sur la façon dont cela est montré. Cela produit une exaltation des spécificités photographiques, c'est-à-dire une exhibition identitaire au lieu d'une dissimulation. L'image montre enfin de quoi elle est faite. Le médium photographique n'est plus abordé comme un moyen neutre de diffuser des informations visuelles mais bien comme une substance propre, capable de susciter des réflexions plurielles dans le champ des arts. Ainsi, les photographies artistiques contemporaines comportent une signature invisible. Le style, l'usage différencié de l'appareil photographique en font un instrument privilégié d'écriture identitaire. Le photographe crée le monde qui se dessine sous ses yeux. Ou plutôt, il en révèle les dimensions cachées.

\section{Photographies de la transparence}

La nouvelle ère numérique s'est accompagnée d'une métamorphose des pratiques artistiques qui, en faisant éclater les cadres, a permis une réflexion en miroir sur les vecteurs de transformations de la photographie. S'en est suivie une révolution ontologique consistant à dévoiler le médium en tant que tel. Non seulement les artistes, aujourd'hui, démantèlent les principes figés par l'argentique, mais plus encore ils reflètent leurs propres innovations. Ils l'exhibent. Un photographe exploitant des images trouvées (sur Internet ou dans certaines archives...) génèrera un jeu avec le spectateur, avide de reconnaître, dans ces œuvres opaques, les références dont elles 
émergent. La poḯtique des œuvres, la nature des images qui la composent, font partie intégrante du travail. Peu importe si elle contient des aspérités, des traces qui trahissent son origine. Tout est fait pour clamer le droit à la différence de l'image, devenue autonome, dissociée du support de la représentation. Est-elle abstraite? Montre-t-elle des sujets anodins? Peu importe. Elle est intéressante pour elle-même. Pourtant, il ne s'agit pas d'une relation spéculaire engageant un face à face entre le visible et le viseur, mais bien d'une émulsion sensible provoquée par le regard déclencheur du photographe. Celui-ci n'est pas étranger à la scène qu'il enregistre. Il en fait partie intégrante. Le mode de restitution ou de falsification de la réalité perçue permet d'affirmer une présence au monde singulière. Toute photographie résulte d'un mélange d'impressions entre le visible et le voyant, au double sens du terme : comme on parle volontiers d'un objet voyant pour signifier qu'il est perçu, mais aussi d'une personne qui est vue. Entre transparence et opacité, le preneur d'images, engagé dans cette relation double à la perception, adapte l'environnement à ses visions subjectives, plus qu'il ne conforme sa vision à celui-ci. C'est parce qu'il s'intéresse à la réflexion sur le médium qu'il va en déployer les possibilités à son paroxysme. S'intéresser à l'appareil d'enregistrement du visible, c'est montrer qu'il capte aussi une part du monde auparavant affranchie de notre regard. Cela revient à en dévoiler les zones disparues de la vision quotidienne. Quel serait l'objet le plus délicat à photographier? La consultation des manuels à destination des praticiens voudrait prouver qu'il s'agit d'un œuf. À cette difficulté portée à son comble, Patrick Bailly-Maître-Grand oppose le comble de la gageure photographique selon lui : saisir l'image d'une boule de verre. La grande parade de la photographie de la transparence se tient dans cette faculté de saisir l'apparition de l'épaisseur du visible dans un objet traversé par le regard. Au-delà de l'observation des diverses formes photographiques mises en place pour la dévoiler, il faut remarquer la révolution ontologique que cet intérêt déclenche. Il ne s'agit plus de parler de la transparence photographique mais de transparence photographiée. Là où la photographie constituait, selon Barthes, un mode de transmission du visible, elle relève, à présent, d'une révélation d'un support invisible. Il est frappant de constater à quel point le motif de la vitre révélée est un élément récurrent dans les pratiques contemporaines. Comparer ces petites épiphanies du quotidien traitées selon différentes perspectives artistiques revient à affirmer une descendance vis-à-vis de certaines théories picturales. Georges Pérec écrit en 1981 qu'« une des limites (un des défis) de la représentation picturale semble être de vouloir se confondre avec l'objet

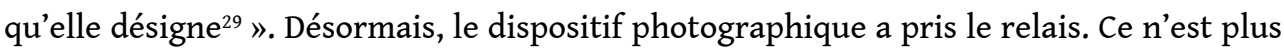
la transparence de son support mais celle de la vitre écran qui s'est muée en objet à contempler. Est-ce pour affirmer sa revanche envers Barthes? À présent, la photographie montre tout et rien à la fois. Lorsqu'un photographe confère une visibilité à une surface transparente, il révèle qu'il n'y a presque rien à discerner, sauf la photographie, la façon dont cela est photographié. Le moyen de voir devient une fin. L'appareil photographique expose ses propres mécanismes comme si la vision découvrait les engrenages de la perception. Voir la vue, regarder un regard, c'est à cette approche axée dans la continuité des truismes de Merleau-Ponty ${ }^{30}$ que nous invite la figuration photographique de la vitre. Dans le même temps, elle nous invite à voir l'invu, regarder un regard aveugle. Faire disparaître l'objet du regard dans l'acte de le voir : telle pourrait être une définition de la disparence photographique. 


\section{1. Définition de la disparence photographique}

Photographier un objet que le regard, habituellement, traverse permet au spectateur de se projeter «au-delà » (trans) de « ce qui apparaît » (parens). Cela génère un arrêt momentané dans la course du regard. Cette démarche constitue dans le même temps une métaphore de la photographie qui, à l'origine, est une vitre sur laquelle se déposent des formes et des matières. Ce serait un moyen de donner à voir la photographie alors que nous choisissons parfois de ne pas la voir. C'est donc une réflexion sur le choix du regard porté en direction d'une chose et non pas d'une autre qu'il convient d'interroger à la lumière de la disparence. Celle-ci est marquée par une répartition tripartite de ses enjeux: la disparence marque à la fois un principe de séparation (dissociation entre ce qui est vu dans l'image et ce que nous voyons dans la réalité), de négation (inadéquation potentielle entre ces deux visions) et d'intensité (métamorphose des perceptions). Trois caractéristiques reflétées par l'image de la vitre qui, une fois photographiée, permet une mise en abyme de ces dimensions. Examiner la vitre revient à parler des conditions de notre perception et à s'inscrire dans le sillage de Maurice Merleau-Ponty qui dément l'existence possible d'une transparence absolue de la vision. Il faut nécessairement faire abstraction de certaines réalités pour en distinguer d'autres. Mais ce partage conduit parfois à exclure de façon dommageable certaines des réalités les plus intéressantes. Les artistes en sont conscients. Aussi, donnent-ils à contempler ces marges et zones de transition où le regard, habituellement, se promène sans s'arrêter. Car la vitre est invisible, dans la réalité. Elle est une condition de passage pour voir ce qui se cache derrière elle. La personne n'ayant pas vu la porte-fenêtre qui sépare deux lieux et s'y engage est devenu l'un des gags les plus éculés des contenus humoristiques favoris des mass médias. La disparence photographique provient alors d'une sorte d'indifférence aux choses, à ce qui nous entoure. Plus qu'un espace où se déploie l'humour, les artistes en ont fait un instrument de sublimation du quotidien, permettant d'examiner la relation du corps sensible à notre environnement afin de déduire le type de complicité existant entre eux :

Le visible autour de nous semble reposer en lui-même, remarque Maurice MerleauPonty. C'est comme si notre vision se formait en son cœur, ou comme s'il y avait de lui à nous une accointance aussi étroite que celle de la mer et de la plage. Et pourtant, il n'est pas possible que nous nous fondions en lui, ni qu'il passe en nous, car alors la vision s'évanouirait au moment de se faire, par disparition ou du voyant ou du visible. [...] quelque chose dont nous ne saurions être plus près qu'en le palpant du regard, des choses que nous ne saurions rêver de voir «toutes nues", parce que le regard même les enveloppe, les habille de sa chair $[. . .]^{31}$.

Montrer la transparence à travers le médium photographique relève a priori d'une gageure du même ordre que s'il s'agissait de montrer notre œil voyant. "Je ne verrai jamais mes rétines, mais si une chose est sûre pour moi, c'est qu'on trouverait au fond de mes globes oculaires ces membranes ternes et secrètes. [...] $]^{32} »$ Cela rejoint le champ des certitudes semblant inscrites en nous de façon indélébile, de sorte que nous n'y prêtons plus d'attention. La révélation de celles-ci les teinte pourtant d'une interprétation nouvelle, faisant basculer nos convictions.

\section{Stances}


Stance $n^{\circ} 4$

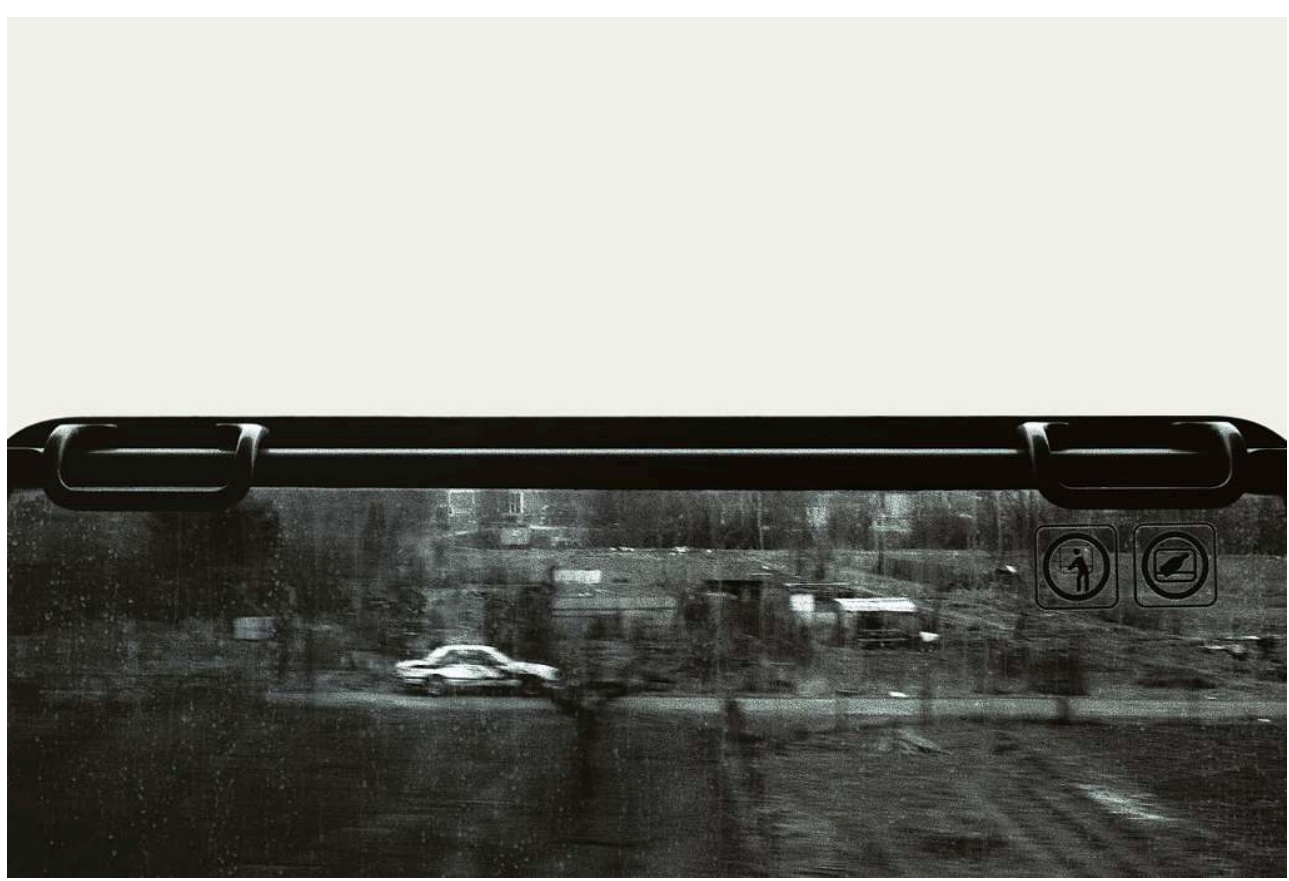

Éric Rondepierre, Ilfochrome sur aluminium, 80 x 120 cm, 1996-1998

\section{Stance $n^{\circ} 6$}

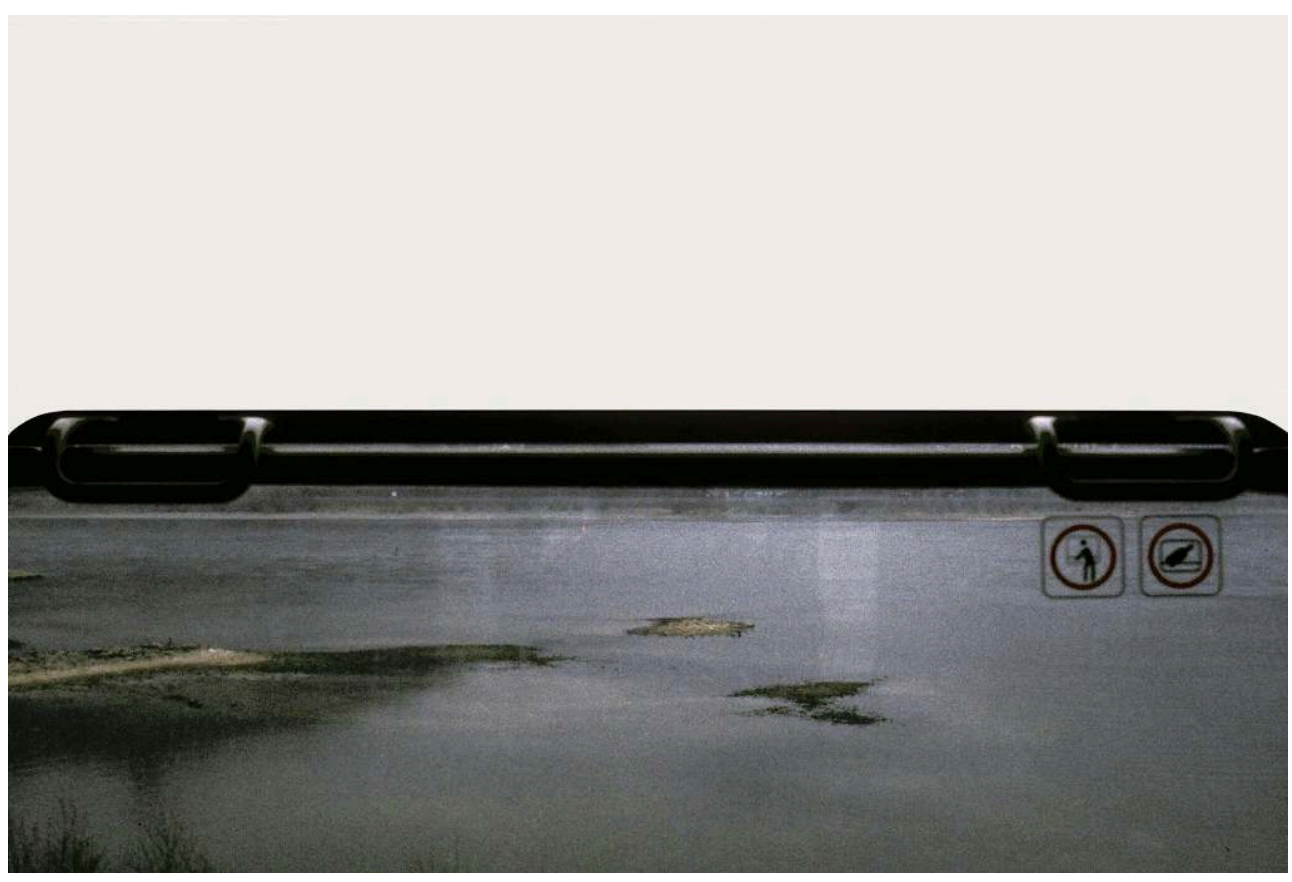

Éric Rondepierre, Ilfochrome sur aluminium, 80 x 120 cm, 1996-1998 
Stance $n^{\circ} 8$

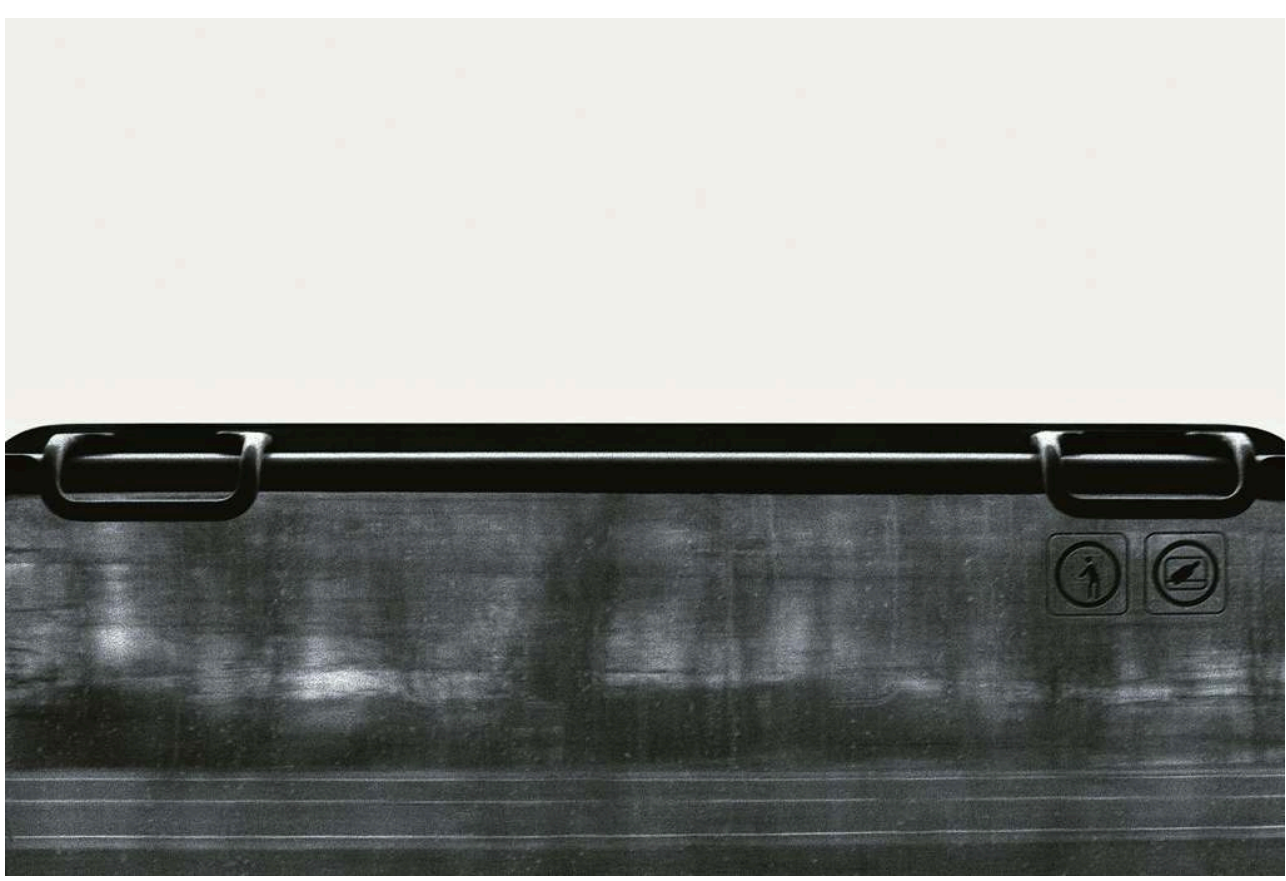

Éric Rondepierre, Ilfochrome sur aluminium, 80 x 120 cm, 1996-1998

\section{Stance $n^{\circ} 10$}

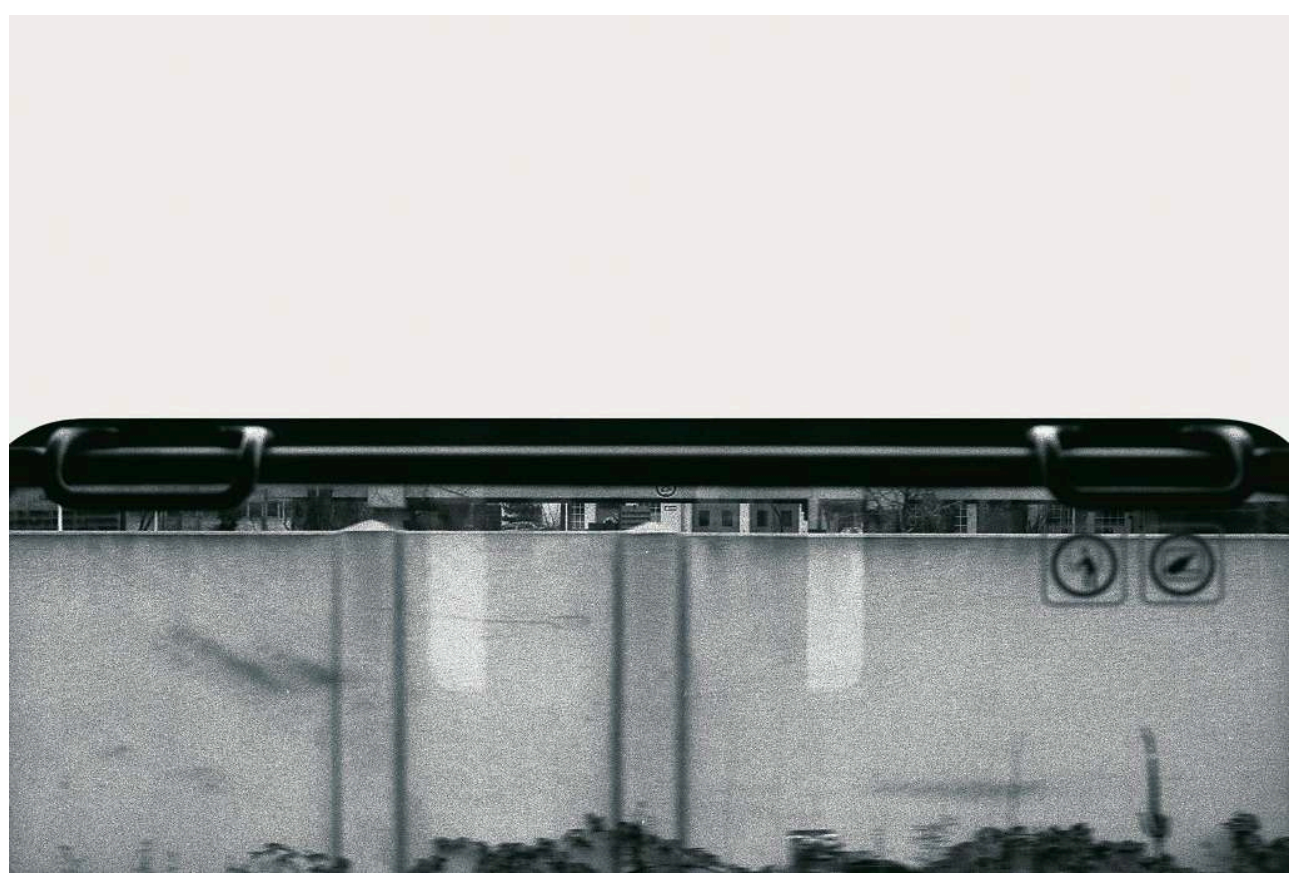

Éric Rondepierre, Ilfochrome sur aluminium, 80 x 120 cm, 1996-1998 


\section{2. Photographies de la disparence}

17 Autour d'une bande visuelle, la disparence photographique permet de s'interroger sur une dialectique des états passagers, remettant en question les théories de la transparence mises au point par Roland Barthes :

Roland Barthes incluait « la vitre et le paysage » dans les dualités qui ne pouvaient que se concevoir, jamais se percevoir. Par son caractère sériel, minimal et néanmoins énigmatique, par cette façon sensible de miner doucement le sol symbolique constitué dans et par cette distinction, Stances entendait bien, sans démonstration, contredire le propos du sémiologue ${ }^{33}$.

Exemple paradigmatique de la disparence photographique, l'œuvre d'Éric Rondepierre permet d'en formuler les enjeux. Le train et la photographie ont une histoire en partie commune. Tous deux semblent évoluer au même rythme, selon des temporalités synchronisées. Leur apparition est concomitante. Puis, leur statut suit un chemin comparable, partagé entre l'invention et la révolution technique accompagnant la fin du XIX ${ }^{e}$ siècle. Ces deux mondes, Éric Rondepierre les a rassemblés dans une série de dix photographies prises depuis la fenêtre d'un train. Elle s'appelle Stances. C'est « une série "à part" "apparemment" ${ }^{34}$ " selon les mots de son auteur. En 1996, Éric Rondepierre est le seul passager de ce train alors qu'il voyage entre Francfort et Cologne. Le plasticien choisit d'exploiter ce concours de circonstances pour tenter une étrange expérience : réunir tous les éléments mis en présence au moment où il regarde par la fenêtre. Ainsi, il tend à rendre compte à la fois de ce qu'il voit, mais aussi des conditions lui permettant d'observer cette coïncidence parfaite entre la barre de la fenêtre et la ligne d'horizon qu'elle dissimule. Inverser les relations déterminant ce qui est vu de ce qui est perçu. Fusionner les expériences perceptives grâce à l'espace préfabriqué de la fenêtre. Modifier la perception en faisant apparaître ce qui était invisible au voyageur (la vitre) et en faisant disparaitre ce qu'il pouvait voir (le paysage). C'est ainsi qu'il bouleverse les codes, rendant lieu d'un renversement des idées reçues. En photographiant la vitre, Éric Rondepierre produit une image négative, comme inversée de la réalité. Michel Guérin relève toute l'ambiguïté de cet objet-métaphore :

Le monde de la transparence n'est pas celui où l'on voit clair enfin, après avoir dissipé des poches d'opacité ; c'est celui qui, ayant usé de tous ses substituts et décidé de se passer désormais de « lieutenance » (de symbolicité, d'analogie), se voit forcé à la situation inédite de voir la vitre elle-même, plus que les objets à travers ${ }^{35}$.

Soudain, le cours de ces univers parallèles s'est arrêté au croisement d'un art où rien ne semble plus pouvoir filer entre les doigts (et les yeux) d'un voyeur devenu acteur de la situation. Quelle était alors cette recherche animant l'esprit du voyageur? Couper, rompre la durée. Rattraper le temps dans sa fuite. Arrêter la course du train. Remonter à la surface des choses. Faire coexister les horizons de la vue avec son contraire... L'obsession d'E. R. semble être de déclencher le signal. Un accident s'est en effet produit sur la rétine de ce passager aux aguets du temps et de son image reflétée. Il a décidé d'embarquer pour ce voyage sans imaginer pour autant que cela génèrerait une telle mue de son regard. Sans doute ne se doutait-il pas alors que la danse des formes derrière la vitre embuée laisserait place à l'errance du regard. Les yeux sont retenus à la paroi de ces tirages ilfochromes, cherchant une sortie au sein des labyrinthes de signes qui se confondent dans la brillance. À présent, notre curiosité se cogne parfois sur les butées qui éraillent l'apparence lisse de la surface; celle-ci n'offrant guère de prises pour cette escalade virtuelle du visible. Le temps délimité des trajets ferroviaires 
aura permis à Éric Rondepierre d'embarquer vers une nouvelle expérience du regard. Le film à contempler? C'était entre chien et loup. Au dehors, le climat rigoureux et froid des contrées allemandes de l'après-midi. L'air extérieur s'engouffrait dans le compartiment à tout vent. La campagne battue par le rythme sourd d'un train déjà ancien. La vitre, entièrement baissée. Le temps d'une route orientée par des rails, des paysages ont défilé. C'est à cet endroit même que le film du visible a pris lieu et place pour se muer dans la disparence. Il n'y avait alors qu'un seul explorateur pour le contempler, le photographier. Découvrant ce qu'il a vu, un indice saute aux yeux : cette vitre, habituellement inexistante à la vue, cette fois, est visible tout en se laissant traverser du regard comme un filtre. Le point de vue est net. Pourtant, quelque chose diffère de la perception réelle que nous en aurions. La succession des images de la nature habituellement engloutie dans les massifs de couleurs et de traits éphémères, apparaît, figée. L'espace intérieur et extérieur ne forment plus qu'un. Le ciel et la terre sont raccordés. Le temps et l'espace, réunis. La lumière et l'obscurité, le monochrome et l'image, l'abstraction et la figuration, le haut et le bas, le plein et le vide, la pensée en son absence, la rigidité des lignes géométriques et le contour vacillant des végétations et autres reliefs escarpés en deçà de l'image, le calme et la vitesse, tout est confondu. L'espace est envahi de signes, différents ou similaires d'une image à une autre. Ils coexistent au sein de la composition, structurée par des jeux de cadres contraints. La partie supérieure de l'image est blanche, vide, alors que les formes occupent toute la partie inférieure. Partagée en deux, la photographie, chaque fois, s'affirme comme un projet complexe, malgré une apparente simplicité de surface.

Photographier la vitre : le sujet n'est pourtant pas nouveau. Serait-ce un clin d'œil aux surréalistes qui se sont employés à immortaliser les vitrines parisiennes, dans la lignée d'Atget? Nous pourrions constituer un répertoire de toutes ces tentatives d'arrêter le regard sur cette surface d'abord conçue pour être traversée par la vue : ainsi pourraiton citer les pratiques différenciées de Jean-Christian Bourcart (Stardust), Patrick BaillyMaître-Grand (Verroteries, etc...) Anna Malagrida (Vista/ Punto), Thibault Hazelzet ou encore Denis Darzacq (Fakestars)... Car chacune de ces démarches met en jeu des problématiques variées, entre technologie du regard et projection psychique du regardeur. Ce qui importe, en effet, réside moins dans l'objet que dans la manière dont il est photographié et de son lien avec la finalité du geste. Dans les Stances d'Éric Rondepierre, il s'agit d'imposer au flux du regard un arrêt. Les choses paraissent avoir été abstraites du compte à rebours propre au mouvement du voyage, comme pour rester immuables, sous vitre (au sens littéral), installées au milieu des cimaises des galeries. Le dos des œuvres semble avoir basculé sur la face de l'image. Une zone blanche paraît franchement répartie et séparée du reste, à moins qu'elle y soit imbriquée. Soudain, la configuration mouvante des choses s'est fixée avec la facilité d'une pensée magique, développée autour d'une formule : « un deux trois soleil » où les enfants sont tenus de stopper leur élan afin de n'être pas pris en faute. La vitre sépare, nie les représentations originelles du visible et intensifie le regard porté sur un objet quotidien. Éric Rondepierre a fait de ces illusions une réalité. Il a arrêté le flux du voyage pour en faire un instant immortel, une éternité déposée subrepticement sur la pellicule. Il a donc conjugué le visible à deux temps différents : au futur, en donnant à voir un support transparent révélé après coup; au passé, en montrant un moment arrêté, arraché au flux du temps pour en être sauvé. Pourquoi la vitre, là, apparaitelle? Quelles raisons déterminent le choix de porter le regard sur un objet qui lui est 
habituellement invisible? Le cours de ce processus de mise en œuvre de la disparence pourrait être expliqué au regard de différents états de l'image à venir.

\section{2. 1. Évaporation du visible}

21 Qui n'a jamais tenté de photographier l'aspect duplice d'un paysage aperçu depuis une fenêtre de train? Montrer l'inéluctable scission du visible. Cadrer un espace déjà restreint par le support de la fenêtre. Arrêter le flux du temps, du train. Jouer sur les oppositions. Les rassembler. Actuellement, l'entreprise paraît insensée : la surface des vitres génère d'importants reflets, si bien que le photographe réalise souvent un autoportrait quand il s'avise de photographier un coucher de soleil perdu derrière les feuillages. Des indices, seulement, marquent la présence des vitres de trains investis par l'artiste entre 1996 et 1998. D'abord, elles font office de masque pour le regard. Il est empêché de voir au loin, au-delà de ce qui est montré par ces rubans en mouvement. Le visible s'évapore, se dissout du champ de la représentation :

Mais c'est dans cette échappée du réel que réside la fascination. Moins le voyageur reconnaît les choses, plus ça va vite, plus il est attentif - mais à quoi ? À la disparition même ?36

Quelque chose manque. Le panorama de notre esprit se trouble à mesure que la figuration disparaît, emportée dans la danse des paysages. Ensuite, des autocollants indiquant des consignes de sécurité génèrent un rythme saccadé au sein des images qui se suivent mais ne se répètent pas. En apparence, c'est une succession monotone de paysages ruraux propres à la Bavière qui étalent leurs plaines et leurs étangs. Les couleurs semblent passées. Le réel s'est teinté d'une couleur étonnante, comme filtrée par la vitre d'où nous percevons le spectacle de la nature. Il y a peu d'objets qui s'offrent au regard. L'attraction du visible s'est dissoute dans sa représentation. La beauté certaine des nuances de ce paysage prend les allures d'un nuancier de gris instables, surmonté d'une zone aveugle. Elle s'est évaporée, disparue dans les mystères du boîtier photographique. À la place des espaces communs, pourtant, des floraisons de signes sont apparues aux yeux du spectateur. Le reflet de l'artiste que nous avions cru absent de l'image est en fait projeté sur ces fractions de temps. Ces frises chronologiques imparfaites n'en montrent qu'un état transitoire. Il a beau être invisible au sens strict du terme, il apparaît en creux au sein des problématiques abordées. Peu à peu, un regard attentif le discerne.

\section{2. 2. Cristallisation de l'Invisible}

23 Si les œuvres d'Éric Rondepierre révèlent parfois « le surgissement de la disparition ", elles parviennent d'autres fois à éveiller un acte d'apparition éteint en direction du spectateur qui semblait inapte à voir ce qu'elles montrent à présent. C'est le cas, ici. Dans cette " œuvre à tiroirs ", la série des Stances fait figure de commutateur, guidant d'abord un principe d'incertitude. Que voyons-nous? La vitre d'un train, une image coupée horizontalement, la cristallisation d'un principe de coupe, la disparition du flux au profit d'une parcelle lacunaire du visible sur laquelle toute l'attention se concentre. Cadrage, dé-cadrage, intérêt perplexe envers un objet quotidien désavoué, qui semblait avoir disparu du champ de notre perception. C'est le principe du hors champ qui intervient comme un déterminisme propre à l'image. Ainsi pouvons-nous parler d'une cristallisation de l'Invisible, sous plusieurs aspects. Au sens physique, elle désigne en effet le phénomène marquant le passage d'une dissolution (liquide) vers la 
solidification des substances, produites par un rapprochement progressif de leurs composants: soit une définition précise du geste accomplit dans les Stances qui permettent de souder le transfuge d'une image en mouvement à son arrêt, sa densification matérielle par le biais de la photographie. Par extension, la cristallisation désigne en effet l'acte de figer, de rester (pour renouer avec l'étymologie latine des Stances). Enfin, elle s'apparente à un terme littéraire né de la plume de Stendhal, renvoyant aujourd'hui au sentiment né de l'imaginaire à partir d'un objet ou d'une personne sur laquelle il se concentre jusqu'à produire un effet de transfiguration. Or, la vue d'E. R. n'a rien à voir, semble-t-il, avec la réalité qui s'est développée lors de ses séjours en train. Tout est métamorphosé dans ces photographies faussement anodines. Les choses, en réalité, ne se partagent pas avec autant de symétrie. La radicalisation formelle avec laquelle ce matériau transparent est envisagé permet d'intercaler des plans de sens en puissance. Mais ils sont pertinemment cachés, dissimulés au regard, sous une masse d'artifices. La disparence est donc une propriété inhérente au médium photographique. Elle est générée par le dispositif de prise de vue.

\section{2. 3. Condensation du visuel}

24 La sobriété des Stances est un leurre auquel répond la synchronie des plans, habilement étagés dans l'espace. Le lieu du visuel s'exprime dans la réception qui est faite d'une image plus que dans l'expérience de la vision. Cette faculté provient d'une expérience de l'après-coup, nous permettant de discerner l'acte même de la vue, selon une perspective nouvelle et différée. Tout ce que nous aurions pu voir en tant que passagers de ce train est présent dans l'espace de représentation. Serait-ce l'œuvre entière d'E. R. déroulée jusqu'en 1996 qui se présenterait à présent sous nos yeux ?

Autant le dire tout de suite: cette série (comme mes séries précédentes) ne ressemble pas à ce qu'elle est, son aspect répétitif et systématique d'apparence renvoie en réalité à un autre champ absent de l'image. Je veux dire par là que sont concentrés en elle des éléments pointant plusieurs niveaux de réalité, mais inclus à l'intérieur de l'image comme dans une sorte de formule, de théorème sensible qui serait à développer. Bien qu'excentrées dans le cheminement de mon travail, ces photos en constituent comme une sorte de relais métaphorique à plusieurs niveaux, qui les replace en son cœur par la bande. Que tout mon dire (en images) se noue dans cette série est ce qu'il faudrait faire apparaître un jour ${ }^{37}$.

Est-ce à dire que les Stances puissent être un concentré artistique à la manière dont le négatif d'une photographie comprend virtuellement toutes les images latentes pouvant être engendrées? Il y a une tension qui règne subrepticement dans ces vues d'apparence sereines, comme portées par une évidence. Or, ce lien est condensé dans la vitre, faisant la charnière perceptive entre tous les cloisonnements de la représentation. Deux zones se partagent l'espace. Cette répartition savante n'est pas anodine. Pourtant, ce cadrage se fait inconsciemment au moment de la prise photographique: « je n'avais pas vu que Stances et Suites suivaient la même structure, précise E. R. Parfois, on est très aveugle sur sa propre production. [...] Je l'ai fait sans m'apercevoir que je cadrais la limite de l'horizon ${ }^{38}$ ». Pourtant, ce ne sont pas des diptyques qui nous sont donnés à voir ici mais bien des images soudant ensemble deux aspects, pour nous en présenter la substance fondatrice : l'acte de séparation entre le ciel et la terre devient la barre de la fenêtre. La ligne d'horizon paraît avoir été dessinée par l'artiste comme un principe corrélé d'opposition et de réunion. La disparence photographique relève d'un oxymore comme d'une évidence. 


\section{2. 4. Sublimation de l'invisibilité}

26 photogrammes trouvés dans les archives des cinémathèques comportaient bien déjà un potentiel de révélation. Le fait de montrer la vitre qui perturbe parfois la vision du paysage s'inscrit dans cette logique. Devenue prétexte à photographier la frontière entre l'intérieur et l'extérieur, elle nourrie un étrange exercice de contemplation où il ne s'agit plus seulement de voir à travers elle mais bien de saisir sa propre matérialité. Devoir de réflexivité par excellence. Arrêter le regard sur ce qu'il traverse habituellement: démarche insolite. Plus que l'expression d'une transparence opaque ayant acquis une visibilité, il s'agit d'une extrapolation du quotidien, à travers un sentiment de troublante singularité. La sublimation est un terme conduisant une double signification: en physique, il désigne le passage d'un corps solide à son état gazeux. Or, les Stances favorisent amplement ces métamorphoses. La matérialité de la vitre semble à présent flotter dans l'apesanteur de la représentation. En ce sens, nous devons alors parler de la transposition d'une «invisibilité » et non plus seulement de l'invisible. La différence est majeure : si la vitre est plus suggérée que véritablement montrée, ce n'est pas forcément elle que l'on voit, mais son action de séparation, son rôle de médiateur. Ici, elle n'est pas seulement pensée pour sa substance. Elle est envisagée, paradoxalement, pour son apparence mais surtout pour sa fonction. Elle est placée là, non pas pour ce qu'elle est mais pour son usage. De nouveau, la vitre devient un transmetteur. La sublimation est aussi un mot employé en psychanalyse pour désigner la transposition d'une pulsion en un état supérieur. Sublimer l'invisibilité de la vitre, cela revient alors à découvrir les raisons ayant motivé ce geste inattendu d'un spectateur distrait ayant voulu faire partager la beauté mitigée d'un spectacle qui se déroulait sous ses yeux. Le film ne correspond à rien qui ait existé. C'est une fiction construite sur l'objectif de l'appareil photographique où se sont reproduites les images de la vitre. Mouvement de retour, effet de mise en abyme. Effet retard ? La beauté de la disparence ne parvient qu'à certains yeux, comme un livre doit trouver ses lecteurs, un film ses spectateurs. C'est souvent lorsque nous regardons dans ses marges qu'elle nous apparait. À d'autres moments, il s'agit de trouver les ouvertures menant à des espaces cachés, seuls garants pour discerner la profondeur d'un message artistique.

\section{2. 5. Disparences en miroir : Éric Rondepierre et Marcel Duchamp}

La disparence est susceptible de produire une invisibilité matérielle de la vitre, envisagée comme métaphore photographique. C'est avec une simplicité illusoire que la fenêtre des Stances répartit les volumes selon deux bandes visuelles horizontales. En acquérant une visibilité, cet objet transparent s'apparente à un trompe l'œil donnant à voir une représentation factice : sa substance se confond avec ce dont elle constitue le cadre; d'une part, le découpage figurant le ciel, vide de sens, d'autre part, l'espace terrestre, semblant avoir éludé ses reliefs. Une séparation franche ne donne pas d'autre choix que d'être prise en main. Des consignes de sécurité font intrusion dans l'image. Le regard du spectateur erre à la surface, tâtonnant pour trouver le chemin organisé d'un horizon. Mais il est tour à tour piégé au sein de l'espace monochrome s'offrant tel une page blanche à (ré)écrire, voire capturé dans les linéaments d'un ruban tacheté, celui d'un paysage. La nature y est progressivement fondue selon des configurations plurielles. Celles de la vitre et du panorama, envisagées comme étendues tacites. La 
densité des masses impose un mouvement centripète, vers le cœur de l'image. Ici, la vitre arrête le regard comme le temps du défilement. Son invisibilité est sublimée au contact de l'espace tridimensionnel du paysage. Tout en prolongeant une esthétique de la transparence, la sobriété formelle des Stances remplit une fonction comparable aux deux vitres qui enserrent Le Grand Verre (1915-1923) de Marcel Duchamp: elles supportent les strates de la représentation en structurant l'espace du "tableau», autrement qualifié de "retard en verre " selon les mots de l'anartiste. Elie During définit cette œuvre comme la "projection tridimensionnelle, elle-même reportée en deux dimensions sur un double panneau de verre, d'une scène invisible à quatre dimensions [où il s'agit de comprendre comment] les différentes dimensions s'articulent les unes aux autres ${ }^{39}$ ». Une définition également transposable à l'œuvre d'E. R. Dans l' opus magna duchampien, l'espace de la représentation est divisé en deux par une barre horizontale, formant deux rectangles d'envergure similaire. Selon Octavio Paz, Le Grand Verre permet l'expression d'une dialectique entre l'œuvre et le spectateur, le visible et l'invisible, le haut et le bas, le mot et l'image. Mais aussi la transparence et ses modulations. Si les rouages mis au point par Duchamp semblent bien rôdés, ils ne sont pourtant pas exempts d'accidents liés au hasard. Les plaques superposées par Duchamp sans autre précaution se fracturent lors d'un transport, en 1926, créant des réseaux de lignes aléatoires venant s'ajouter aux géométries peintes : la transparence véhicule des impressions nouvelles. La vitre est alors donnée à voir en tant que surface d'inscription. Les fractures ainsi générées composent des géométries sous forme de ready-made, comme la barre horizontale des Stances produit une dichotomie contrainte au sein de l'espace de représentation. Celle-ci permet la mise en œuvre paradoxale d'une quatrième dimension sous forme bidimensionnelle (photographique). Expression d'un hyperespace. Évocation d'un mouvement sans cesse reconstruit par les métamorphoses du paysage. Le propos des Stances échappe à son apparente géométrie euclidienne grâce à la vitre qui s'interpose. Là où l'œuvre de Duchamp entretenait une relation en miroir avec la "boîte verte", c'est à la boîte noire que la série d'Éric Rondepierre fait directement allusion. Seule la vitre est importante: "une restriction sur le sujet s'exprime à l'aide d'autres moyens [de négation], notamment les termes seul et seulement ${ }^{40} »$. Et ceux qui la découvrent se heurtent à une démonstration solitaire. Les preuves résident dans le cadre énonciatif des Stances: le passager de l'image a beau composer un dialogue entre les deux parties de la photo, sa parole n'intervient pas directement, comme si le hasard devait déterminer les morceaux choisis du paysage à sa place, à l'instar des lignes accidentelles du Grand Verre. L'environnement spatial est celui d'une faille temporelle, un arrêt prolongé dans la course du temps. Le projet de cette chambre avec vue rejoint alors celui de Fresh Widow : il s'agit dans les deux cas de surfaces aveugles sur lesquelles le regard, désormais, s'arrête. Dans cette œuvre de Duchamp, le verre transparent a laissé place à l'opacification du spectacle, avec de brillants carreaux de cuir. La fenêtre ainsi murée rejoint la représentation de Matisse dont elle semble inspirée. Dans Porte-fenêtre à Collioure (1914), les préceptes du peintre sont appliqués à la lettre: le noir y apparaît comme le vecteur d'une lumière éblouissante. Si Marcel Duchamp définit le goût comme la « répétition de toute chose déjà acceptée ${ }^{41} »$, alors l'enchâssement des références pourrait en être le garant. Comme Flaubert, il paraît scruter inlassablement un « sujet invisible » comme disparu, réduit à l'expression du mot. Le Grand Verre, qui est-il ? Mais c'est surtout vers Raymond Roussel qu'il se tourne quand il s'agit de revendiquer une filiation: Impressions d'Afrique, 
en premier lieu, auquel il faut sans doute ajouter un long poème en vers plus confidentiel intitulé La vue, s'ouvrant sur un incipit révélateur :

La vue est une très fine photographie

Imperceptible, sans doute, si l'on se fie

À la grosseur de son verre dont le morceau

Est dépoli sur un des côtés, au verso

Mais tout enfle quand l'œil plus curieux s'approche

Suffisamment pour qu'un cil par moments s'accroche ${ }^{42}$

"Photographies » de "l'imperceptible », les Stances affirment la scansion répétée d'un visible échappé du champ de son appartenance d'origine. Ainsi révèlent-elles les caractéristiques inhérentes à la disparence photographique (séparation, négation et intensité). La transparence de la vitre, comme celle de la photographie, est métamorphosée. L'ontologie du médium trouve lieu et place dans la topologie. L'« évaporation du visible », la "cristallisation de l'invisible », la "condensation du visuel » et la "sublimation de l'invisibilité » représentent les avatars d'une réflexion sur le regard et la perception de son objet, comme la vitre devient un sujet de réflexion privilégié, permettant l'apparition magique de lanternes cachées.

\section{BIBLIOGRAPHIE}

Barthes Roland, La Chambre claire, Paris, Seuil, 1980.

Bourdieu Pierre, Un art moyen, Essai sur les usages sociaux de la photographie, Paris, Minuit, 1965.

Boutaud Jean-Jacques, «La Transparence, nouveau régime visible », in Transparence et communication, Jean-Jacques Boutaud (dir.), MEI, nº 22, Paris, L'Harmattan, 2005.

Didi-Huberman Georges, «L'Indice de la plaie absente, Monographie d'une tache », Traverses, $\mathrm{n}^{\text {os }}$ 30-31, « Le secret », mars 1984.

Didi-Huberman Georges, La Ressemblance par contact, archéologie, anachronisme et modernité de l'empreinte, Paris, Minuit (Paradoxe), 2008.

Freud Sigmund, « Le moi et le ça » [1923], S. Jankélivitch (trad.), Paris, Payot, 1968.

Guérin Michel (dir.), La transparence comme paradigme, Aix-en-Provence, publications de l'université de Provence, 2008.

Jouffroy Alain, « Éric Rondepierre, visionneur », in Éric Rondepierre, Paris, Léo Scheer, 2003.

Krauss Rosalind, « Notes sur l'index », in L'Originalité de l'Avant-garde et autres mythes modernistes, Paris, Macula, 1993.

Merleau-Ponty Maurice, Signes, Paris, Gallimard, 1960.

Merleau-Ponty Maurice, «L'entrelacs - le chiasme », in Le Visible et l'Invisible, Paris, Gallimard, 1964.

Modiano Patrick, Rue des Boutiques Obscures, Paris, Gallimard, 1978.

Pérec Georges, L'œil ébloui, Paris, Hachette, 1981. 
Récanati François, La Transparence et l'énonciation : pour introduire à la pragmatique, Paris, Seuil, 1979.

Riegel Martin, Pellat Jean-Christophe, Rioul René, Grammaire méthodique du français, Paris, PUF, Quadrige, 2001.

Rondepierre Éric, Carnets, Paris, La Revue littéraire/Léo Scheer, 2005.

Rouillé André, « L'énonciation photographique », Paris, Les Cahiers de la photographie, nº 5, 1982.

Roussel Raymond, La vue, Paris, Jean-Jacques Pauvert, 1963.

Schaeffer Jean-Marie, L'image précaire, Du dispositif photographique, Paris, Seuil (Poétique), 1987.

Walton Kendell, "Transparent Pictures: On the Nature of Photographic Realism", Critical Inquiry, 11/2, 1984, p. 246-277.

\section{NOTES}

1. Jean-Jacques Boutaud, "La Transparence, nouveau régime visible", in Transparence et communication, Jean-Jacques Boutaud (dir.), MEI, n 22, Paris, L'Harmattan, 2005.

2. Roland Barthes, La Chambre claire, Paris, Seuil, 1980, p. 18.

3. Les traducteurs envisagent « la transparence sonore du lexique » en termes de pourcentages, afin de définir des paliers de compréhension favorisant le passage d'une langue à l'autre.

4. François Récanati, La Transparence et l'énonciation: pour introduire à la pragmatique, Paris, Seuil, 1979.

5. Rosalind Krauss, " Notes sur l'index ", L'Originalité de l'Avant-garde et autres mythes modernistes, Paris, Macula, 1993.

6. Cf. note des éditeurs (J. Gilbert-Rolfe / R. Krauss / A. Michelson) ouvrant le nº 1, October, 1976.

7. Ibid., p. 64.

8. Patrick Modiano, Rue des Boutiques Obscures, Paris, Gallimard, 1978.

9. Pierre Bourdieu, Un art moyen, Essai sur les usages sociaux de la photographie, Paris, Minuit, 1965.

10. Kendell Walton, "Transparent Pictures: On the Nature of Photographic Realism", Critical Inquiry, 11/2, 1984, p. 246-277.

11. Roland Barthes, La Chambre claire, p. 16.

12. Ibid.., p. 17.

13. Ibid., p. 22.

14. «Parmi [les photographies] qui avaient été choisies, évaluées, appréciées, réunies dans des albums ou des revues, et qui avaient de la sorte passé au filtre de la culture, je constatais que certaines provoquaient en moi de menues jubilations, comme si celles-là renvoyaient à un centre tu, un bien érotique ou déchirant, enfoui en moi-même (si sage en fût apparemment le sujet) ; et que d'autres, au contraire, m'indifféraient tellement qu'à force de les voir multiplier, comme de la mauvaise herbe, j'éprouvais à leur égard une sorte d'aversion, d'irritation même ", Ibid., p. 34.
15. Ibid., p. 42.
16. Ibid., p. 65.
17. Ibid., p. 119.
18. Ibid., p. 120.
19. Ibid., p. 115.

20. Sigmund Freud, « Le moi et le ça » [1923], S. Jankélivitch (trad.), Paris, Payot, 1968, p. 18.

21. Georges Didi-Huberman, La Ressemblance par contact, archéologie, anachronisme et modernité de l'empreinte, Paris, Minuit (Paradoxe), 2008.

22. Ibid.., p. 187. 
23. Ibid.., p. 190.

24. Nous nous référons ici au texte original (en français) : Georges Didi-Huberman, «L'Indice de la plaie absente, Monographie d'une tache ", paru dans Traverses, $n^{\text {os }} 30-31$, « Le secret ", mars 1984.

25. Allusion à l'ouvrage éponyme de Daniel Arasse.

26. Georges Didi-Huberman, «L'Indice de la plaie absente, Monographie d'une tache », paru dans Traverses, $\mathrm{n}^{\text {os }}$ 30-31, « Le secret », mars 1984, p. 153.

27. André Rouillé, "L'énonciation photographique », Paris, Les Cahiers de la photographie, nº 5 , 1982, p. 31.

28. Jean-Marie Schaeffer, L'image précaire, Du dispositif photographique, «Avertissement ", Paris, Seuil (Poétique), 1987, p. 9.

29. Georges Pérec, L'œil ébloui, Paris, Hachette, 1981, n.p.

30. «Le penser pense, la parole parle, le regard regarde » (Maurice Merleau-Ponty, Signes, Paris, Gallimard, 1960, p. 5).

31. Maurice Merleau-Ponty, "L'entrelacs - le chiasme », in Le Visible et l'Invisible, Paris, Gallimard, 1964, p. 171.

32. Ibid.., p. 190.

33. Éric Rondepierre, Carnets II, Paris, Léo Scheer, 2005, p. 124.

34. Propos recueillis lors d'un entretien avec Éric Rondepierre, 2007.

35. Michel Guérin (dir.), La transparence comme paradigme, Aix-en-Provence, publications de l'université de Provence, 2008.

36. Alain Jouffroy, «Éric Rondepierre, visionneur », in Éric Rondepierre, Paris, Léo Scheer, 2003, p. 117.

37. Éric Rondepierre, Carnets, Paris, La Revue littéraire/Léo Scheer, 2005.

38. Propos recueillis lors d'un entretien avec Éric Rondepierre, 2007.

39. Elie During, « Mondes virtuels et quatrième dimension : Duchamp, artiste de science-fiction ", revue Alliage, $\mathrm{n}^{\circ} 60$, juin 2007, version numérique transmise par l'auteur.

40. Martin Riegel, Jean-Christophe Pellat, René Rioul, Grammaire méthodique du français, Paris, PUF, Quadrige, 2001, p. 413.

41. Marcel Duchamp, entretien avec James Hohnson Sweeney, 1955, in Duchamp du signe, Paris, Flammarion, 1994, p. 175-185.

42. Raymond Roussel, La vue, Paris, Jean-Jacques Pauvert, 1963, p. 2.

\section{RÉSUMÉS}

De la transparence de la photographie à la photographie de la transparence, se joue l'avènement d'un nouveau paradigme contemporain. En conjuguant l'analyse du noème barthésien sous sa forme décomposée à une esthétique de la réception qui arrête le regard sur le médium habituellement traversé par celui-ci, il s'agit d'examiner le passage de la transparence à la disparence photographique, de l'image pensive (vitrine) à l'image réflexive (miroir). 
INDEX

Mots-clés : contemporain, index, photographie

Personnes citées : Rondepierre (Éric), Duchamp (Marcel), Barthes (Roland)

\section{AUTEUR}

\section{MURIEL BERTHOU CRESTEY}

Docteur en esthétique, thèse sur « L'Invisible : un révélateur pour la photographie contemporaine " 